\title{
THE INSULIN-GLUCOSE TOLERANCE TEST. A MODIFIED PROCEDURE FOR THE DETECTION OF HYPOGLYCEMIA UNRESPONSIVENESS IN PITUITARY AND ADRENAL INSUFFICIENCY
}

\author{
BY FRANK L. ENGEL AND JAMES L. SCOTT \\ (From the Department of Medicine, Duke University School of Medicine, \\ Durham, North Carolina)
}

(Received for publication July 5,1949 )

In 1941, Fraser, Albright, and Smith (1), described the application of three carbohydrate tolerance tests to the diagnosis of disturbances in the endocrine control of metabolism. These were the glucose tolerance test, the insulin tolerance test, and the glucose-insulin tolerance test. When properly employed and interpreted, these tests are of considerable value. However, in practice, the insulin tolerance test has often proven disappointing in those situations in which it should be most valuable, i.e., conditions characterized by hypoglycemia unresponsiveness. Characteristically in adrenal and pituitary insufficiency, and less so in hyperinsulinism, the response to insulin is somewhat exaggerated and the spontaneous rise in blood sugar after hypoglycemia is much retarded. This is clear from the data of Fraser et al., when the individual curves for patients with hypopituitarism and Addison's disease are compared with the mean of the normals, or conversely, when cases of myxedema or anorexia nervosa are compared to the mean of the panhypopituitary group. However, in practice, we have found considerable overlapping between the curves from cases of panhypopituitarism and Addison's disease and those from cases of myxedema and anorexia nervosa and with the normals, which makes individual curves difficult to evaluate. While varying degrees of adrenal and pituitary insufficiency may occur in myxedema and anorexia nervosa, it is doubtful whether all the abnormal insulin tolerance tests in these conditions have this origin. Furthermore, the intravenous injection of insulin into patients with panhypopituitarism or Addison's disease is attended by a very definite risk of severe or even fatal hypoglycemia. For this reason, Fraser et al. recommended that one-half or one-third the usual dose of insulin be given to patients seriously suspected of suffering from these conditions. Often this results in equivocal responses and the necessity for repeating the test. Even in presumably normal individuals the symptoms of hypoglycemia may sometimes be sufficiently distressing as to make it desirable to terminate the procedure by giving glucose.

The purpose of this communication is to describe a modification of the insulin tolerance test, the insulin-glucose tolerance test, which was designed to overcome some of the disadvantages of the former test. It is based on the observation by Somogyi (2) that in normal individuals the administration of glucose 30-60 minutes after insulin, i.e., during hypoglycemia, resulted in the development of greater hyperglycemia than the administration of the same amount of glucose without insulin. Thus, glucose magnified the normal homeostatic response to hypoglycemia: This response is presumably mediated by the hormones of the anterior pituitary, adrenal medulla and adrenal cortex. It was found in the present study that compared to normal individuals, patients with adrenal and pituitary insufficiency did not have the capacity to raise their blood sugar levels rapidly from hypoglycemic levels when given glucose. This made for a considerably greater separation between this group of patients and normals and other pathological conditions than is achieved by the insulin tolerance test, although it did not eliminate overlapping. Furthermore, and of very great importance, the administration of glucose shortly after insulin considerably decreased the dangers from prolonged hypoglycemia in patients with adrenal or pituitary insufficiency. Although the test has its greatest value in the hypoglycemia unresponsive group, it was applied to a variety of other conditions for comparative purpose.

\section{METHODS}

Normal medical students and hospitalized patients were the subjects for these tests. All hospitalized patients were 
on the regular hospital diet for at least three days prior to the test. This contains approximately 2,600 calories (protein 100, fat 130, and carbohydrate 250). The normal medical students ate their regular diets which were of variable composition. Although it is recognized that rigid control of the diet is important in tests of this sort, this precaution is so rarely followed faithfully in practice that it was felt desirable to compare the results.under the less favorable circumstances under which the tests are apt to be carried out.

\section{PROCEDURE}

The subject is fasted overnight. Venous blood is withdrawn in the morning and 0.1 unit of regular insulin per $\mathrm{Kg}$. body weight injected intravenously. In individuals weighing $40 \mathrm{Kg}$. or less U40 insulin should be diluted tenfold with saline in order to administer an accurate dose. At 30 minutes or when the first significant symptoms of hypoglycemia appear, depending on which is first, 0.8 gram of glucose per $\mathrm{Kg}$. body weight is given by mouth. Additional blood samples are taken at 60,90 , 120 , and 180 minutes from the initial sample. For comparative purposes the blood sugar levels are recorded as per cent of the initial blood sugar, which is taken as 100 per cent.

\section{RESULTS}

Figure 1 records the mean curve ( \pm standard deviation) of the insulin-glucose tolerance test in 30 subjects : 20 medical students and 10 hospitalized patients who were well nourished and had no evidence of organic disease. Although the medical students were on variable diets there was no statistically significant difference in their response

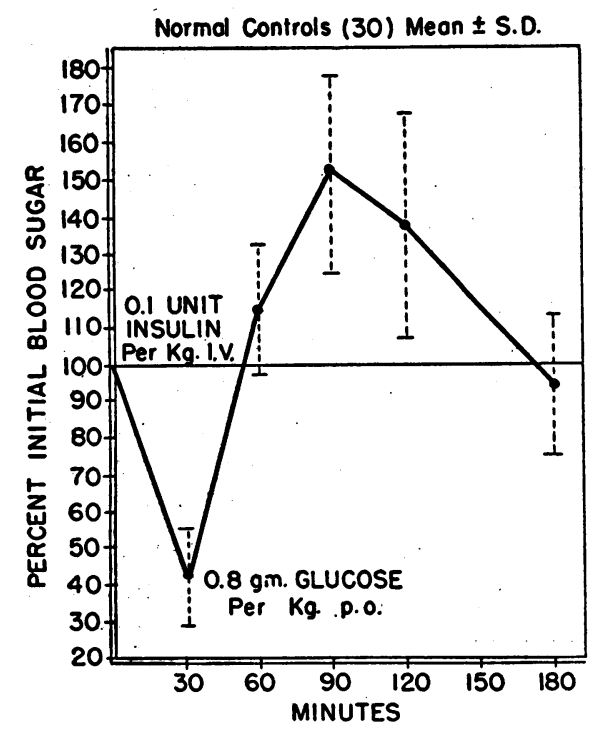

Fig. 1. Mean Insulin-Glucose Tolerance Curve \pm S'tiandard Deviation for 30 Normal Individuals

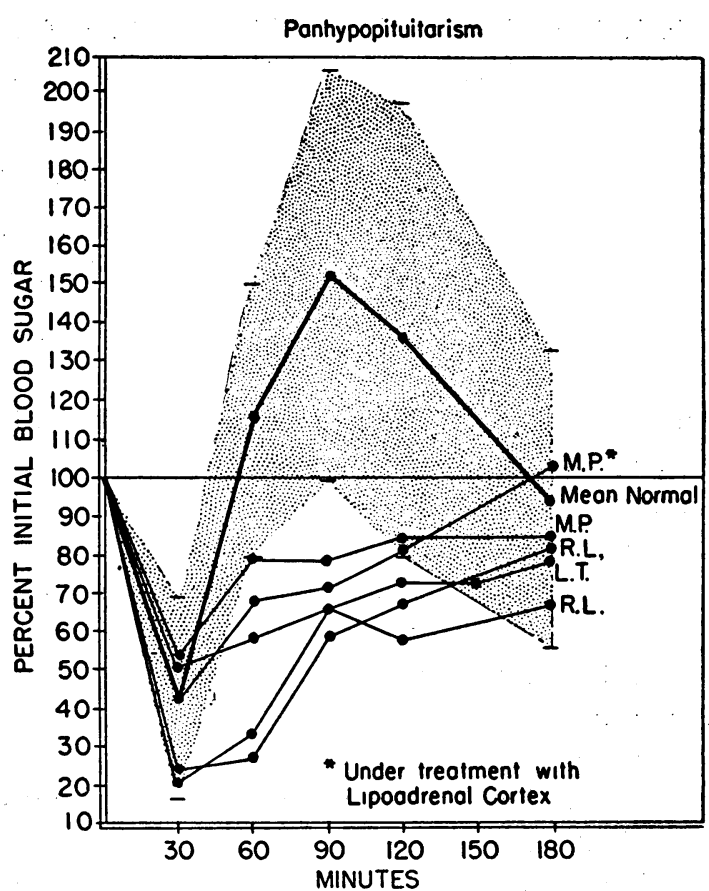

Fig. 2. Insulin-Glucose Tolerance Curves from Three Patients with Panhypopituitarism

The shaded area in this and subsequent figures represents twice the standard deviation of the mean normal and should include the curves from 97 per cent of normal individuals. The probability of a curve falling outside this area being normal is very small.

compared to the hospitalized controls. The two groups were therefore pooled for determination of the standard deviation of the mean. A characteristic biphasic pattern is apparent, with a prompt rise of the blood sugar to a mean above the starting level 30 minutes and a peak 60 minutes after glucose. In no single case was there a failure to exceed the initial value at either 30 or 60 minutes after glucose.

Figure 2 records five curves after insulin and glucose, plotted as per cent of the initial blood sugar, from three patients suffering from panhypopituitarism. The diagnosis in each case was based on classical history, physical and laboratory findings, including abnormally low urinary excretion of 17 -ketosteroids and gonadotrophins. M. P. had a craniopharyngioma and was slightly undernourished; R. L. had a chromophobe adenoma which was removed surgically, and was moderately obese; and L. T. was a classical example of postpartum pituitary necrosis with pituitary cachexia. These five curves, as well as subsequent ones are 
plotted against a background of the mean normal curve surrounded by an area representing tzrice the standard deviation of the mean of the normal. The probability of any curve falling outside the shaded area being normal is small, since theoretically the area should include over 97 per cent of all normals, while half the area would include 67 per cent of all normals. In actual fact no curve and only one single point of any of the 30 curves from normal subjects studied fell outside of the shaded area, indicating that the limits of normal set by this procedure are indeed liberal. This method of plotting, therefore, graphically illustrates the significance of each curve compared to normal, and gives a fairer indication of the validity of the test than is obtained by the usual custom of comparing them simply to the mean normal. Note that the shapes of the curves from the hypopituitary patients are very different from the normals. The fall in blood sugar 30 minutes after insulin is not significantly different from normal but the normal sharp . rise after glucose is absent, being replaced by a gradual rise toward the fasting value. The blood sugars at 60,90 and 120 minutes are all

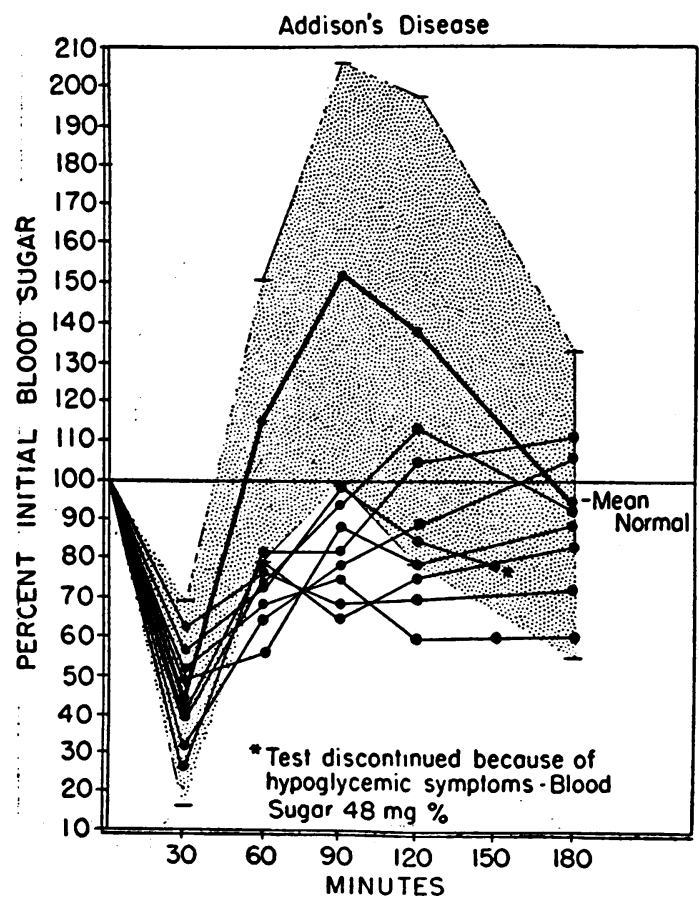

Fig. 3. Insulin-Glucose Tolerance Curves from Eight Patients with Addison's Disease

Three:additional cases studied since this chart was prepared have shown similar abnormal curves. either outside or just at the lower edge of the theoretical normal range. Although the number of cases is small they are consistent and, as will be noted below, were little changed by various types of therapy.

Figure 3 shows a set of curves from eight patients with Addison's disease compared to the mean normal with twice the standard deviation. The decreased or delayed response to glucose is again apparent as in the hypopituitary cases. All curves had one or more points outside of the shaded area, particularly at 60 and 90 minutes, leading to a flattened appearance to the curve. In contrast to the normals where everyone exceeded the initial value at either 30 or 60 minutes, or both, none of the Addison's disease curves had reached this level at these times. One curve (marked with an asterisk) fell almost within the normal, including a peak at 90 minutes. However, the fasting blood sugar in this patient was low, making the representation of the curve in terms of per cent of the initial value misleading. The actual blood sugar values corresponding to the points on the chart were $59,33,45,58,50$ and $48 \mathrm{mg}$. per cent. Indeed, the test was discontinued at 150 minutes because it was apparent that the patient was having a return of hypoglycemic symptoms. It is clear that with a low initial blood sugar more significance must be attached to the absolute blood sugar levels than the per cent changes. Since the chart was drawn three additional cases of Addison's disease have been studied with similar results.

Nine patients in whom the diagnosis of Addison's disease or panhypopituitarism was suspected but ruled out by other procedures were studied (Figure 4). With one exception, the curves all were within the normal range. The exception (marked with an asterisk), who gave a borderline response with a slow rise to a peak at 180 minutes and with the 90 minute value significantly lower than normal, was a case of acromegaly who was tested two weeks after removal of a pituitary tumor and had a stormy postoperative course. Preoperatively she had had amenorrhea and low urinary gonadotrophins, but no other evidences of hypopituitarism. Unfortunately no other pituitary function tests were carried out at the same time as the insulin-glucose tolerance test, but her subsequent course has not been such as to sug- 


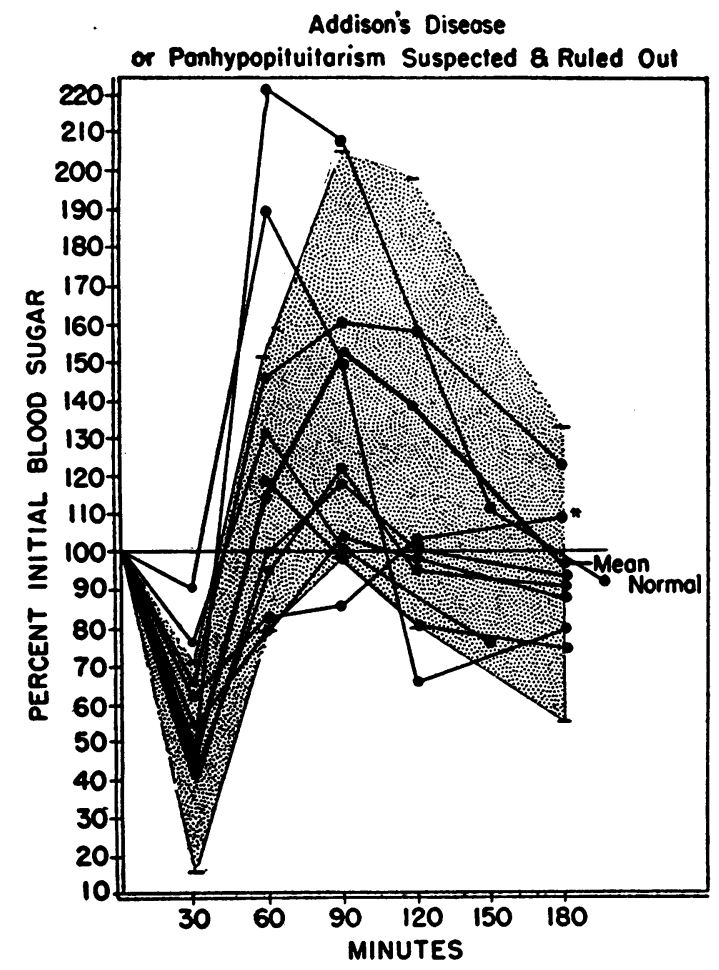

Fig. 4. Insulin-Glucose Tolerance Curves from Nine Patients in Whom the Diagnosis of Adrenal or Pituitary Insufficiency Was Considered but Ruled OUT

The curve marked with an asterisk was from a case of acromegaly tested two weeks after removal of a pituitary tumor.

gest the development of panhypopituitarism. It is clearly possible that she may have been suffering from a temporary panhypopituitarism at the time the test was done, but since there is no proof of this, she has been put in the above group.

Six patients with marked malnutrition on a psychogenic basis, and three with malnutrition from organic disease, all weighing from 57 to 75 pounds, were studied. They all were females and four met the criteria for the diagnosis of anorexia nervosa. Figure 5 shows the results of the insulin-glucose tolerance test in this group. Two patients had definitely delayed rises after glucose but reached peaks of 115 and 130 per cent at 90 and 120 minutes, falling to close to the initial value at 180 minutes. The shapes of the curves differentiated them from the hypoglycemia unresponsive groups. The delayed rise suggested poor absorption from the gastrointestinal tract without the concomitant metabolic disturbance seen in pituitary or adrenal insufficiency. One case had a curve indistinguishable from the adrenal or pituitary insufficiency group. This was a markedly malnourished 37 year old married woman with amenorrhea and vomiting on a psychogenic basis in whom adrenal and pituitary disease was ruled out by other procedures. One patient with anorexia nervosa who showed an abnormal response to the insulin tolerance test had a normal insulin-glucose tolerance test. In view of the fact that patients with extreme malnutrition such as occurs in anorexia nervosa may in fact show varying degrees of pituitary insufficiency, it is surprising that there were not more abnormal curves in this group. Three patients similarly malnourished, but with organic disease showed essentially normal responses.

Five of six patients with myxedema yielded normal curves after insulin and glucose (Figure 6). Two of those five fell within the normal range but had delayed peaks, suggestive of poor absorption. One patient ( $T$.) showed a response identical with the hypopituitary and hypoadrenal pa-

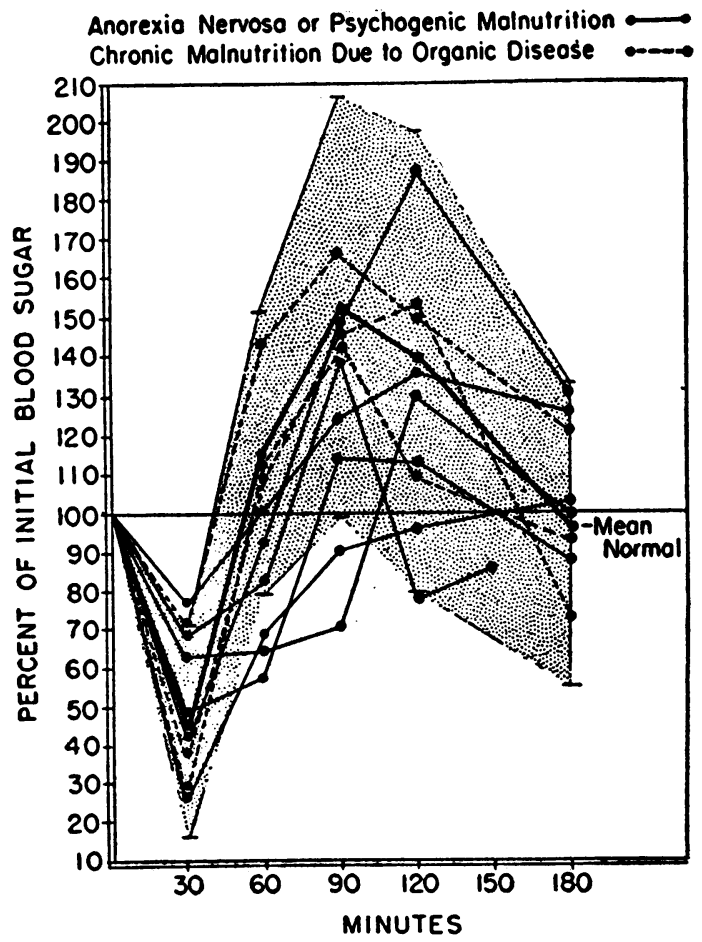

Fig. 5. Insulin-Glucose Tolerance Curves in Nine Patients with Severe Malnutrition, Three Because or Organic Disease, Four with Anorexia Nervosa and Two with Psychogenic Vomiting 


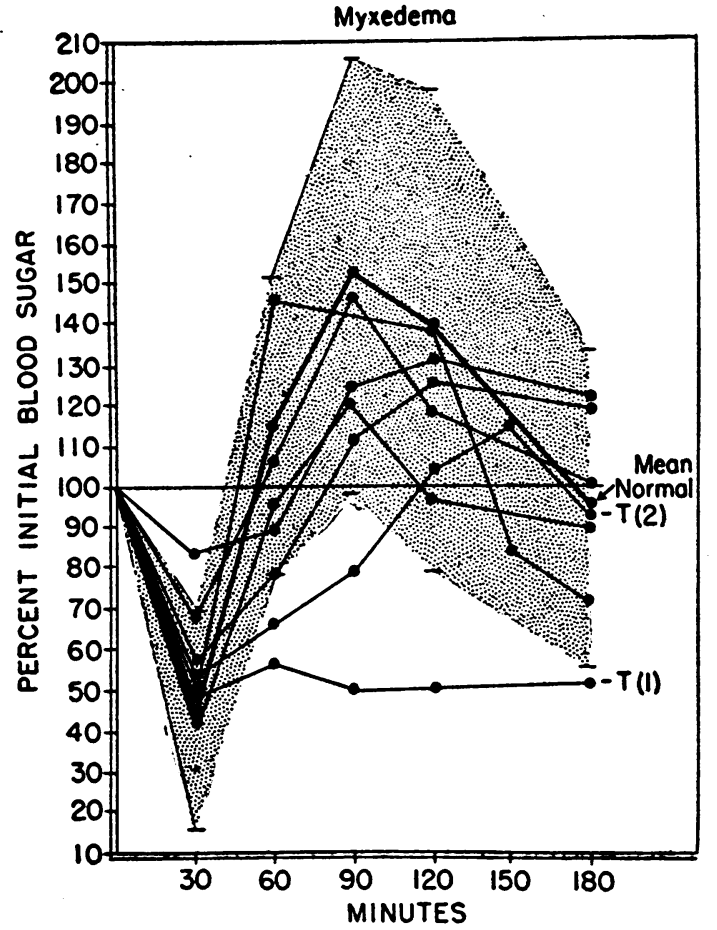

Fig. 6. Insulin-Glucose Tolerance Curves in Six Patients with Myxedema

The curves marked $T(1)$ and $T(2)$ were done before and after four weeks of thyroid therapy. This patient had findings suggestive of adrenal cortical insufficiency before therapy.

tients. Since the rate of absorption of carbohydrate from the intestinal tract is impaired in severe hypothyroidism the test was repeated with the same dose of glucose intravenously as $80 \mathrm{ml}$. of a 50 per cent solution of glucose. The blood sugar fell to 61 per cent of the initial value 30 minutes after insulin, was 119 per cent of the initial value 30 minutes after glucose, 53 per cent 30 minutes later, 67 per cent 60 minutes later, and 81 per cent 150 minutes after glucose. The precipitous fall of the blood sugar after glucose suggests that poor absorption was not the explanation of the defect. The patient was a 58 year old single man with severe myxedema of probably 40 years duration. He was stunted in growth, had a typically cretinoid facies, and was very much mentally retarded. He came to the hospital in severe congestive failure with marked peripheral edema. BP 80-110/60-80. His testes were small and soft but his penis was not underdeveloped. Axillary and pubic hair were lacking, beard growth present but sparse. His BMR, uncorrected for edema was - 50 per cent; serum cholesterol $340 \mathrm{mg}$. per cent; hemoglobin 8.5 grams, RBC 2.9 million; and WBC 2,500 with 50 per cent polymorphonuclears, 3 per cent esosinophils, 1 per cent basophils, 32 per cent lymphocytes and 14 per cent monocytes. Serum sodium was $130.4 \mathrm{mEq}$. per liter; chloride, $89.6 \mathrm{mEq}$. per liter; potassium, $5.3 \mathrm{mEq}$. per liter. The Kepler test was positive. Because of the severity and long duration of the myxedema in this case it seemed possible that the evidences suggestive of adrenal and pituitary insufficiency, including the insulin-glucose test, were secondary to the thyroid deficiency rather than the reverse. For this reason he was treated with thyroid extract alone. On this he showed dramatic improvement in all spheres with subsidence of congestive failure, rise in blood pressure, loss of 20 $\mathrm{Kg}$. in weight, restoration of serum electrolytes to

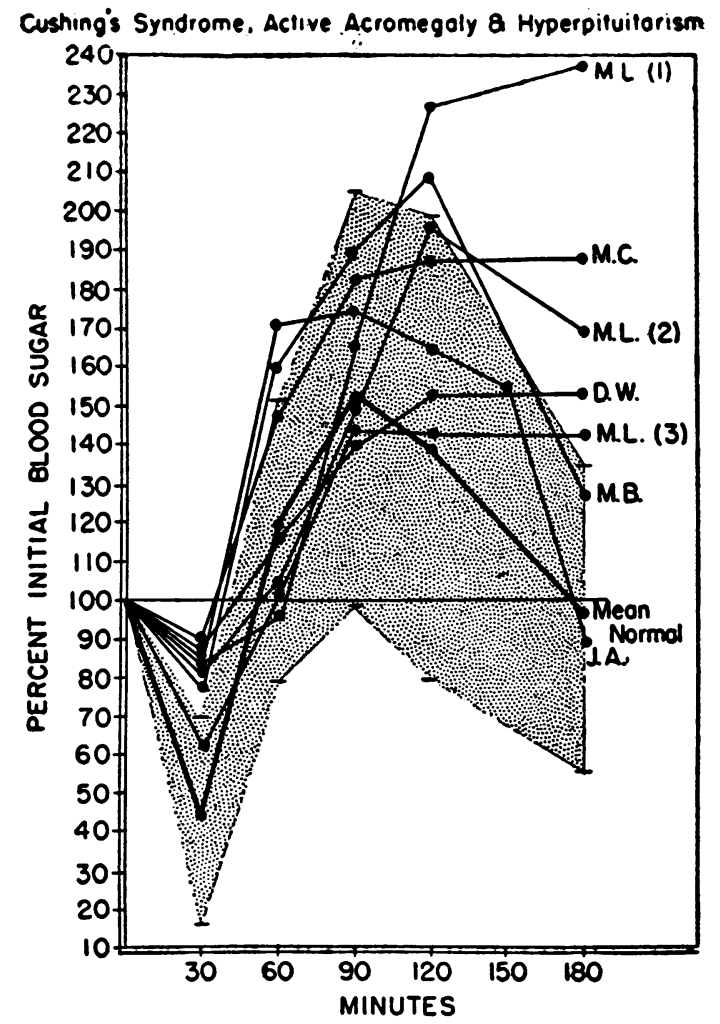

Fig. 7. Insulin-Glucose Tolerance Curves in Cushing's Disease (M. L., M. B.), Acromegaly (D. W. and M. C.) and a Mixed Tumor of the Pituitary (J. A.)

M. L. was tested preoperatively (1), three weeks (2), and eight weeks (3) after removal of an adrenal adenoma. 
normal and fall in BMR and serum cholesterol. After four weeks of therapy his insulin-glucose tolerance test (T2) was considerably improved. The quick response to thyroid therapy alone strongly supported the interpretation that the above changes were secondary to thyroid insufficiency, since one would not anticipate reversal of the electrolyte and other changes suggestive of adrenal insufficiency by thyroid if this were pituitary myxedema.

Five cases of adrenal and pituitary hyperfunction were studied (Figure 7). D. W. and M. C. had active acromegaly; M. B., Cushing's disease due to adrenal hyperplasia; M. L., Cushing's disease due to adrenal adenoma; and J. A., a mixed tumor of the pituitary. In all cases the curves were characterized by a significantly impaired fall in the blood sugar after insulin and by either or both exaggerated rises or delayed falls after glucose. The three tests on M. L. were carried out preoperatively, and three and eight weeks after removal of adrenal cortical adenoma. These curves are shown in contrast to the hypoglycemia unresponsive group, and are as to be expected in patients with insulin resistance and impaired glucose tolerance. From the diagnostic standpoint it is questionable whether the insulin-glucose tol-

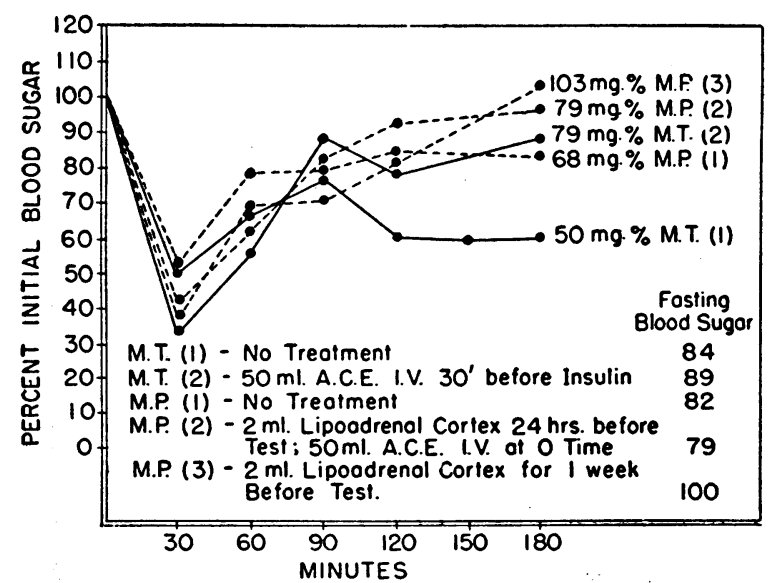

Fig. 8. Effects of Treatment with Adrenal Cortical Extract on the Insulin-Glucose Tolerance Curves in Addison's Disease (M. T.) and PanhypoPII UITARISM (M. P.)

Initial and final blood sugar levels are recorded. Although the curves are the same, the actual blood sugar levels are higher in M. P. after treatment -with lipoadrenal cortex. erance test offers any advantages over other well accepted procedures used in these conditions.

\section{Studies on the nature of the defect in hypoglycemia unresponsiveness}

A series of observations designed to clarify the nature of the defect in these patients was carried out on the hypoglycemia unresponsive group. From the results it is clear that the normal biphasic response to insulin and glucose represents a complex set of mechanisms for the maintenance of homeostasis which is lacking in the absence of the adrenal and pituitary glands and not easily reproduced by available therapeutic measures.

Figure 8 demonstrates that the normal response is not restored either by chronic or acute therapy with adrenal cortical extract, at least in the doses used here. Patient M. T. had Addison's disease. Curve 1 was recorded during a time when she was receiving no therapy. Thirty minutes before the administration of insulin for the second test 50 $\mathrm{ml}$. of Upjohn's adrenal cortex extract were injected intravenously over about 10 minutes. Although the blood sugar levels rose to slightly higher levels the response was not normal and not significantly improved over the response with no therapy. Three tests were carried out on patient M. P., a case of panhypopituitarism due to a craniopharyngioma. Curve 1 shows the result with no previous therapy, Curve 2 , that after receiving $2 \mathrm{ml}$. of Upjohn's lipoadrenal cortex 24 hours before the test and $50 \mathrm{ml}$. of aqueous adrenal cortex extract at the same time as the insulin. Test 3 was done after the patient had been under treatment with $2 \mathrm{ml}$. of lipoadrenal cortex daily for one week. None of these treatments had any effect on the shapes of the curves. However, treatment with lipoadrenal cortex for one week resulted in a significantly higher fasting blood sugar and a higher absolute final blood sugar. Even after a short fast when liver glycogen should be adequate, $50 \mathrm{ml}$. of adrenal cortex extract did not improve the response, as is seen in Figure 10. The secretion of the equivalent of $50 \mathrm{ml}$. of commercial extract by the adrenal glands in response to hypoglycemia, therefore, cannot by itself be responsible for the prompt rise in blood sugar seen in normal individuals after glucose administration during hypoglycemia. 
Since the immediate response to hypoglycemia is usually mediated by the adrenal medulla, which may be destroyed in Addison's disease and whose function may be impaired in hypopituitarism (3), the effects of epinephrine were studied in two patients (Figure 9). W. W., a case of Addison's disease due to adrenal tuberculosis, was given $0.007 \mathrm{mg}$. of epinephrine per $\mathrm{Kg}$. body weight in $200 \mathrm{ml}$. of saline intravenously over 45 minutes prior to the injection of insulin. The blood sugar rose from 63 to $94 \mathrm{mg}$. per cent and then fell progressively after insulin, even after glucose was administered. In this case, pretreatment with epinephrine caused further impairment of the subsequent response to insulin and glucose. This worsened response is probably due in part to further depletion of liver glycogen by epinephrine, and in part to secretion of endogenous insulin in response to the rising blood sugar unopposed by the normal antagonists to insulin. L. T., a case of panhypopituitarism due to post-partum pituitary necrosis was given the same dose of epinephrine simultaneously with the insulin and over a 30 minute interval. Although a normal type of curve was not achieved, the blood sugar rose to a considerably higher level by 180 minutes than it had prior to therapy. Epinephrine alone also apparently cannot reproduce the normal response to insulin and glucose.

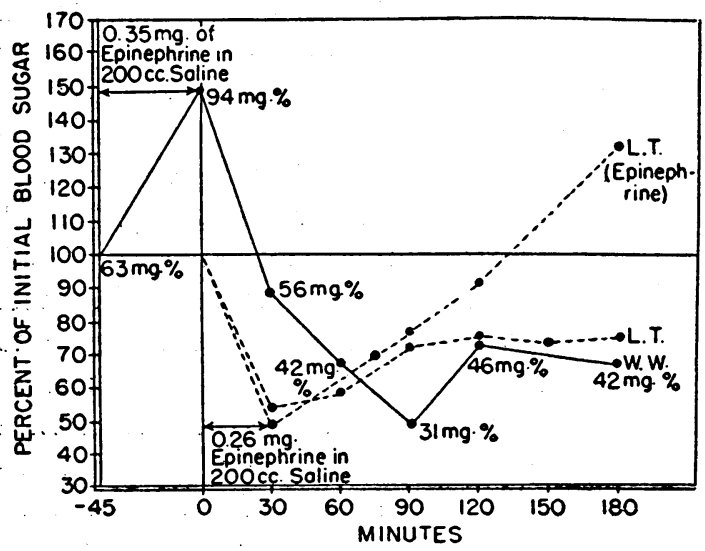

Fig. 9. Effects of Intravenous Epinephrine Before and After Insulin Administration on the InsulinGlucose Tolerance Curves in Addison's Disease (W. W.) and Panhypopituitarism (L. T.)

Epinephrine before the insulin resulted in an impaired response to glucose. Epinephirine after insulin was followed by a late rise in blood sugar.

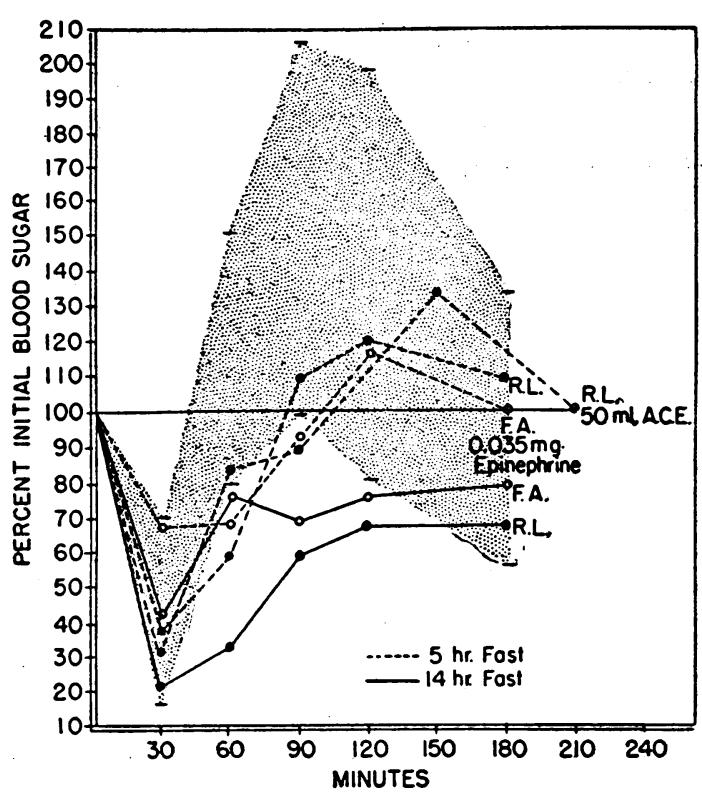

Fig. 10. Effect of a Short Fast (Five Hours) on the Insulin-Glucose Tolerance Test in Panhypopituitarism (R. L.) ANd Addison's Disease (F. A.)

The curves after a five hour fast are nearer the normal than those after a 14 hour fast and are not altered by either $50 \mathrm{ml}$. of adrenal cortex extract or $0.007 \mathrm{mg}$. of epinephrine per $\mathrm{Kg}$. injected intravenously during the $\mathbf{3 0}$ minutes following insulin.

An adequate level of the liver glycogen must be one important factor in making possible a rapid rise in blood sugar after hypoglycemia. Since the liver glycogen is presumably depleted in the fasting state in panhypopituitary and adrenal insuffcient patients compared to normal, the effect of repletion of the liver glycogen by feeding was studied (Figure 10). Patient R. L., a case of panhypopituitarism due to a chromophobe adenoma, was first tested in the fasting state and found to have a markedly abnormal response. Retested on another occasion five hours after breakfast, a delayed, but almost normal response was achieved. Injection of $50 \mathrm{ml}$. of Upjohn's adrenal cortex extract during the first 10 minutes did not improve the response after the short fast. F. A., a case of Addison's disease similarly fasted (Figure 10), was given $0.007 \mathrm{mg}$. of epinephrine per $\mathrm{Kg}$. intravenously after the insulin. Although the response in this patient was significantly improved compared to his curve after a 14 hour fast, it was not better than R. L.'s after a five hour fast without epinephrine. These results suggest that 
an inadequate supply of liver glycogen is one of the more important factors in preventing the normal return of the blood sugar after insulin and glucose in the panhypopituitary individual.

\section{DISCUSSION}

As described here, the insulin-glucose tolerance test is simple to perform and appears to be useful in the diagnosis of conditions characterized by hypoglycemia unresponsiveness. Among 40 curves from patients with diseases commonly confused with adrenal or pituitary insufficiency (including 10 determined since the charts were drawn) two (one patient with psychogenic vomiting and one with anorexia nervosa) showed hypoglycemia unresponsiveness after oral glucose. Two other patients (a case of severe myxedema and a case of acromegaly two weeks after removal of a pituitary tumor) had abnormal curves but also had other abnormalities suggestive of adrenal and pituitary insufficiency. Further studies are necessary to determine whether more patients in the chronically malnourished group might show hypoglycemia unresponsiveness. The occurrence of varying degrees of pituitary insufficiency in anorexia nervosa is well known. It would be surprising if the insulin-glucose tolerance test did not also reflect this tendency.

The insulin-glucose tolerance test has the advantage over the insulin tolerance test in being associated with considerably less risk, provided the proper precautions are taken. In contrast to the latter, the precautions necessary to avoid accidents due to hypoglycemia do not modify the response to the test or occasion its repetition. As long as the blood sugar has begun to fall before the glucose is administered, the characteristic response may be expected. In this clinic there was one serious hypoglycemic reaction in a patient with Addison's disease due to the fact that the observer delayed giving glucose until 30 minutes despite the fact that hypoglycemic manifestations were obvious at 20 minutes. In one patient with Addison's disease mild hypoglycemic symptoms occurred at 150 minutes. The secondary fall in blood sugar which may occur in some patients makes it advisable to have the patient eat immediately after the conclusion of the test.

The chief usefulness of this procedure should be in the screening out of Addison's disease and pan- hypopituitarism suspects, particularly in clinics where the newer and more specialized diagnostic techniques are not available. A prompt and substantial rise in the blood sugar after insulin and glucose can probably be taken as fairly strong evidence against adrenal or pituitary insufficiency. In the clinically more obvious cases of insufficiency, particularly when more specific procedures are available, there should be relatively few occasions where either the insulin or the insulinglucose tolerance test is indicated for the final diagnosis unless serious doubt still exists. As with all procedures of this type, the results can only be interpreted in terms of the total clinical picture and cannot be used as an isolated diagnostic procedure.

The precise mechanism of the disturbed response to insulin and glucose in adrenal and pituitary insufficiency is not yet completely understood. In panhypopituitarism the disturbance in carbohydrate metabolism is due to the combined effects of the deficiency of the adrenocorticotrophic hormone, and hence the adrenal cortex, and of the growth hormone. In clinical practice the precise roles of each of these deficiencies in accounting for the changes in carbohydrate metabolism remain obscure. It is generally believed that the adrenal insufficiency is the more important factor in determining the sensitivity to insulin. In experimental animals it is known that a decrease in glycogen stores in liver and muscle, a low fasting blood sugar and a hypersensitivity to insulin occur in the hypophysectomized animal after insulin injection. Administration of potent adrenal cortical steroids will overcome all of these abnormalities except the decrease in muscle glycogen which is restored by treatment with the pituitary growth factor (4). There is no information on the effect of purified growth hormone on the response to insulin of the hypophysectomized animal treated with adrenal cortical extract. In the present state of our knowledge, therefore, the response to insulin and glucose in the test under consideration can only be considered a rough measure of combined pituitary and adrenal function and in no way distinguishes between abnormalities arising in one or the other gland. Nor is the test as carried out here a specific indicator of adrenal or pituitary function. The ability to elevate the blood sugar above normal after the administration of 
glucose during hypoglycemia is dependent on a variety of factors, not all of which are mediated solely by the adrenal cortex and anterior pituitary gland. In order to elevate the blood sugar promptly after ingestion of glucose, there must be : (1) a rate of utilization of carbohydrate at this time which is less than the rate of supply of glucose from the gastrointestinal tract and from the liver by glycogenolysis and gluconeogenesis; (2) a normal ability to absorb glucose from the intestinal tract; (3) an adequate level of liver glycogen to meet the requirements of (1); and (4) a normal ability to release glucose from liver glycogen, either by discharge of epinephrine or any other method of hepatic glycogenolysis. In hyperinsulinism utilization of carbohydrate may be excessive so that condition (1) may not be fulfilled. It may be anticipated that the insulinglucose tolerance test would be abnormal during the phase of active secretion of large amounts of insulin in this condition. In conditions associated with marked disturbance in intestinal absorption, such as sprue, a delayed rise in blood sugar might be anticipated. No such cases have been studied. In myxedema, carbohydrate absorption is delayed. This may be responsible for the slower rise in blood sugar noted in two of our cases. In untreated panhypopituitarism probably all four factors are disturbed and enter into the abnormal response. The rate of carbohydrate utilization is increased above normal, intestinal absorption is decreased, glycogen stores are diminished during fasting and glyconeogenesis is deficient, and according to de Bodo (3), the hepatic glycogenolytic response to epinephrine is deficient. The same factors are at play in adrenal insufficiency, except that there is no good evidence that carbohydrate utilization is increased except during stress, and there is generally destruction of the adrenal medulla and hence epinephrine deficiency. The experimental data described in this report suggest that the level of the liver glycogen must be one of the more important factors in determining the abnormal response to the insulinglucnse tolerance test in pituitary and adrenal insufficiency. If the immediate decrease in glucose utilization in the periphery which follows hypoglycemia (5) is due to adrenal cortical secretion, this effect must be due to an amount of hor- mone greater than that contained in $50 \mathrm{ml}$. of commercial extract. This amount of extract produced no significant effect in either five or 14 hour fasted adrenal and pituitary insufficient patients. Admittedly the normal adrenals might put out considerably more hormone, or a more impressive response might be achieved by the use of more potent cortical steroids such as 11-dehydro-17-hydroxycorticosterone.

\section{SUMMARY}

1. A procedure is described for detecting hypoglycemia unresponsiveness which carries less risk than does the conventional insulin tolerance test and magnifies the differences between the normal and the hypoglycemia unresponsive patients.

2. The mean response in 30 normal individuals to the intravenous administration of 0.1 unit insulin per $\mathrm{Kg}$. body weight followed by 0.8 gram of glucose per $\mathrm{Kg}$. body weight by mouth $30 \mathrm{~min}$ utes later consisted of a fall in the blood sugar to approximately 45 per cent of the initial value followed by a prompt rise to a peak of 152 per cent at 90 minutes and a slow fall to the initial value by 180 minutes.

3. In each of 13 curves from three patients with panhypopituitarism and eight with Addison's disease, there was a statistically significant decrease in the response to glucose after insulin, yielding relatively flat curves compared to the normals.

4. In eight patients in whom Addison's disease or hypopituitarism were suspected but ruled out by other procedures the insulin-glucose tolerance tests were not significantly different from normal. In one case in which hypopituitarism was not definitely ruled out, the response was equivocal.

5. In a group of nine patients with severe emaciation, six due to psychogenic causes and three due to organic disease, one patient with psychogenic vomiting fell clearly into the hypoglycemia unresponsive group and one with anorexia nervosa was borderline.

6. Five of six patients with myxedema had normal responses. The sixth fell into the hypoglycemia unresponsive group and showed considerable improvement after thyroid therapy. Data were presented to suggest that this patient might have had adrenal insufficiency secondary to longstanding thyroid failure. 
7. Five patients with pituitary and adrenal overfunction syndromes showed a decreased fall in the blood sugar after insulin and either an exaggerated or a prolonged rise after glucose.

8. Neither epinephrine nor cortical extract therapy restored to normal the response in the hypoglycemia unresponsive group. Performance of the test after only a five hour fast yielded a near normal response. The significance of the liver glycogen level in this test is emphasized.

\section{ACKNOWLEDGMENT}

We are indebted to Dr. Haywood M. Taylor and the Staff of the clinical biochemistry laboratory of the Department of Biochemistry for blood sugar determinations on 22 of the 88 insulin-glucose tolerance curves reported bere.

\section{BIBLIOGRAPHY}

1. Fraser, R. W., Albright, F., and Smith, P. H., The value of the glucose tolerance test, insulin tolerance test, and glucose-insulin tolerance test in the diagnosis of endocrinologic disorders of glucose metabolism. J. Clin. Endocrinol., 1941, 1, 297.

2. Somogyi, M., A paradoxical effect of insulin on glucose assimilation. Fed. Proc., 1948, 7, 190.

3. de Bodo, R. C., Slater, I. H., Weisberg, H. F., and Prescott, K. F., Adrenaline hyperglycemia in hypophysectomized dogs. Fed. Proc., 1948, 7, 26.

4. Russell, J. A., The relationship of the anterior pituitary to the thyroid and the adrenal cortex in the control of carbohydrate metabolism. Essays in Biology, in honor of Herbert M. Evans. University of California Press, 1943.

5. Somogyi, M., Studies of arteriovenous differences in blood sugar. III. Effect of insulin administered intravenously in the postabsorptive state. J. Biol. Chem., 1949, 179, 217. 